\title{
Segmentation and Recognition of Petroglyphs Using Generic Fourier Descriptors
}

\author{
Vincenzo Deufemia and Luca Paolino \\ Department of Management and Information Technology \\ University of Salerno \\ Via Giovanni Paolo II, 132, 84084 Fisciano(SA), Italy \\ \{deufemia, lpaolino\}@unisa.it
}

\begin{abstract}
In this paper we present an approach for the segmentation and recognition of petroglyphs from images of rock art reliefs. To identify symbols we use a shape descriptor derived by 2-D Fourier transform, which is independent to scale and rotation, and robust to shape deformations. The efficacy of the algorithm has been validated by testing it with scenes and test images extracted from the archeological site located in Mount Bego (France). The results have been compared with those obtained by other descriptors.
\end{abstract}

Keywords: Symbol recognition, Fourier transform, petroglyph reliefs.

\section{Introduction}

Petroglyphs are symbols carved into a rock surface by prehistoric people. Their preservation is one of the primary objectives for many government institutions, which are trying to identify and archive them for future generations. As an example, the Indiana MAS project, which is granted by the Italian Ministry of University and Research, aims to provide a framework for the digital protection and conservation of rock art natural and cultural heritage sites [1112.

An important task in the digital preservation of rock carvings is represented by the detection of petroglyph symbols and their classification according to their shape and position on the rock panel. For example, Figure 1 shows a black and white relief extracted from a rock panel [9]. The image depicts many petroglyph symbols. Some of them can be recognized at first look, especially corniculate and daggers, even though they are split in several parts. Other symbols are overlapped or connected with lines. The cracks in the rock introduce further challenges in symbol identification process.

In order to support archaeologists in these activities, in the recent years several approaches have been proposed to automate the detection of pecked regions from rock panels [15, the classification of petroglyphs based on their shape [14, and the retrieval of similar petroglyphs from archives of petroglyph images [21]. In this paper we propose a new approach for the segmentation of petroglyph scenes and recognition of petroglyph symbols. The recognition process is based on an Enhanced version of Generic Fourier descriptors (EGFD) [18, a descriptor

A. Elmoataz et al. (Eds.): ICISP 2014, LNCS 8509, pp. 487-494, 2014.

(C) Springer International Publishing Switzerland 2014 


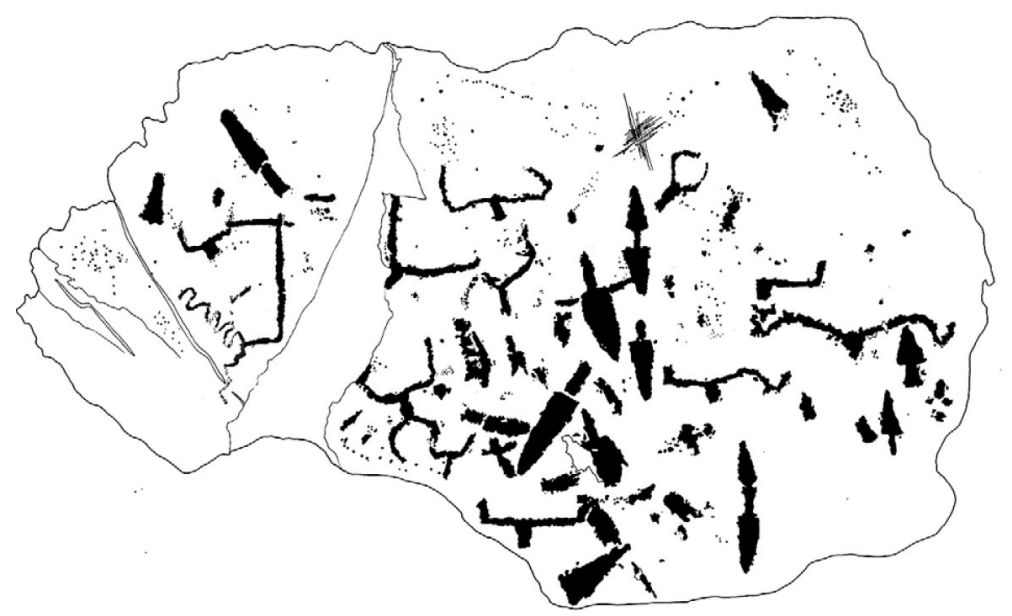

Fig. 1. A relief captured from a rock panel on the Mount Bego 9 ]

invariant to translation, rotation, and scaling, and robust to deformations. The algorithm we propose examines each isolated part of the scene extracted from a rock panel by combining them with the closer ones in order to obtain the more likely combination.

The proposed recognition system has been evaluated on a dataset of $53 \mathrm{com}-$ plex scenes each containing 22 petroglyph symbols on average. Symbols have been compared with a dataset of petroglyphs collected and interpreted by de Lumley's team on Mount Bego 4. The dataset consisted of 94 images from 10 classes. Experimental results show that the proposed GFD-based algorithm outperforms the recognizers based on Image Deformation Model (IDM) [10] and Generalized Hough Transform (GHT) 21] descriptors, both in terms of recognition accuracy and speed.

The contribution of this paper is threefold: (a) it proposes the use of extended GFD to classify petroglyphs; (b) it describes how to combine GFD-based recognizer with segmentation and selection algorithms for decomposing complex scenes, last but not least, (c) it evaluates the performance of the approach on real scenes.

The paper is organized as follows. Section 2 provides a discussion of related work. Section 3 presents an overview of the proposed approach. Section 4 focuses on the experiment we performed to analyze the performance of the approach. Section 5 concludes the paper.

\section{Related Work}

So far the recognition of petroglyph symbols has received little attention from the graphics recognition community [21. One of the first work in this field was mainly for catalogue purposes [16]. Here authors studied petroglyphs in terms of lengths 
of parts of animal bodies, and relations among petroglyphs of several regions. Successively, in 2006, Takaki et al. [17. proposes some methods to characterize shapes of the petroglyphs and the properties of the group they belong to by expressing them through elementary symbols and statistics of petroglyph groups.

Zhu et al. proposed a distance measure and algorithms based on the GHT which allow data mining of large collections of rock art images [21]. In [14] Seidl and Breiteneder presented some preliminary results about their approach for the segmentation of rock art images. They also presented in [15] an approach for the identification of pecked regions from digital photos. The output of this algorithm corresponds to the digital reliefs taken as input by the proposed approach. In 7. we presented an algorithm which leverages on the IDM. Differently from the GFD approach presented in this paper, it is time consuming and rotate dependent. The petroglyph recognizer proposed in [5] fuses an image-based recognizer with a fuzzy visual language parser [3] in an attempt to combine the knowledge of how the petroglyphs look (appearance) with the knowledge of how they relate (recurrent patterns). Finally, visual analytics has been used to support the interpretation of new archaeological findings [6] and the analysis of wall painting degradations [20].

\section{The Proposed Approach}

In this section we present the approach proposed for the segmentation of petroglyph scenes into pecked regions, their clustering and recognition as petroglyph symbols. The recognition process exploits the generic Fourier descriptor (GFD), which is extracted by applying a modified polar Fourier transform on a rectangularized polar shape image [19]. To make GFD invariant to rotation and scaling its features are normalized based on the radial and angular frequencies. Moreover, the similarity between two shapes is measured with the Euclidean distance between their feature vectors. The GFD descriptors have been improved in [18] through a shape normalization performed before the extraction of the features. In particular, the rotation and scale normalization of the input images allows to obtain an enhanced generic Fourier descriptor (EGFD) more robust to skewed, stretched, and distorted images.

Figure 2 depicts the proposed approach. For applying the EGFD algorithm we create the sign dataset $S$, namely for each petroglyph symbol we extract the corresponding feature vector. We will use this dataset to compute the similarity between the components extracted from the scenes and the petroglyph symbols.

The segmentation and recognition process consists of four steps:

1. The scene to be analyzed is cleaned from the elements which are not part of petroglyph symbols, namely thin lines, rock breaks, little points, and so on. To this aim, we convert the grayscale image to a binary image and apply a median filtering.

2. The obtained image is segmented by extracting the connected components. Those with fewer than 50 pixels are removed from the image. As an example, 


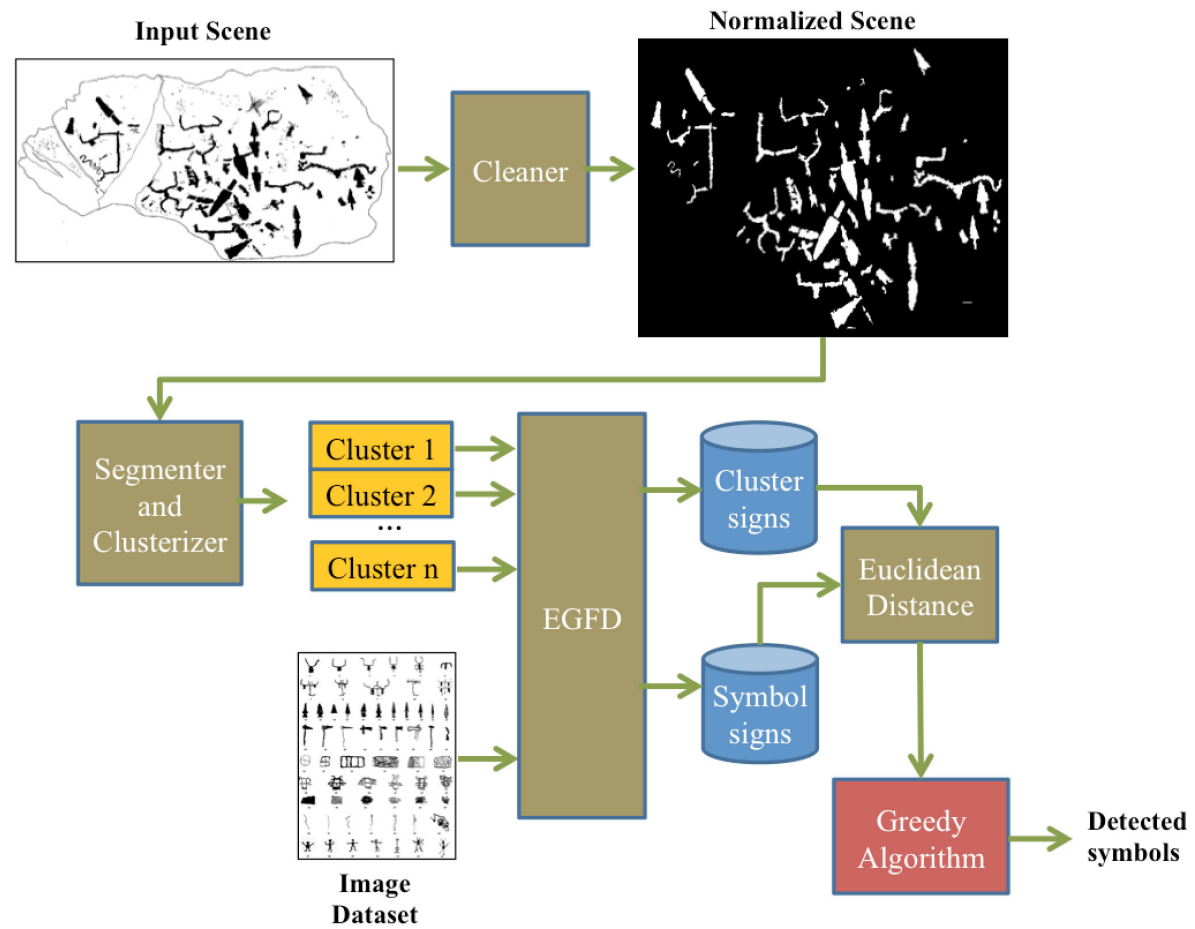

Fig. 2. The segmentation and recognition process

Figure 3(a) shows the 84 connected components identified from the cleaned image derived from the scene of Figure 1.

3. The connected components are grouped into clusters based on the Euclidean distance among them. The result of this step is a set $L$ of clusters of connected components.

4. In the last step, a greedy algorithm selects the elements from $L$ based on the EGFD distances among them. In particular, starting from an element $e$ in $L$, the greedy approach creates the candidate symbols by grouping $e$ with the elements in $L$ having the smallest EGFD distance from $e$, and evaluates their similarity with a petroglyph symbol through the values produced by the EGFD algorithm. As an example, Figure 3(b) depicts the candidate symbols selected by the algorithm.

\section{The Experiments}

The experiment we performed aims to evaluate the approach in terms of efficiency and accuracy. 


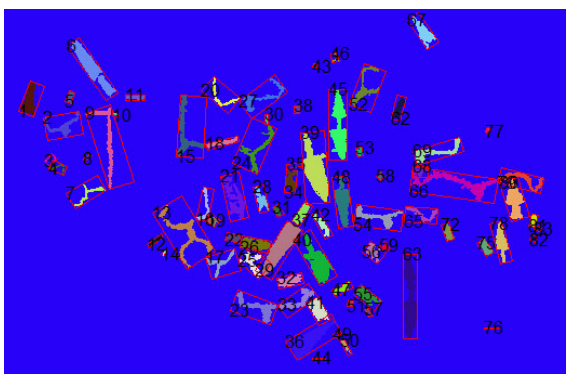

(a)

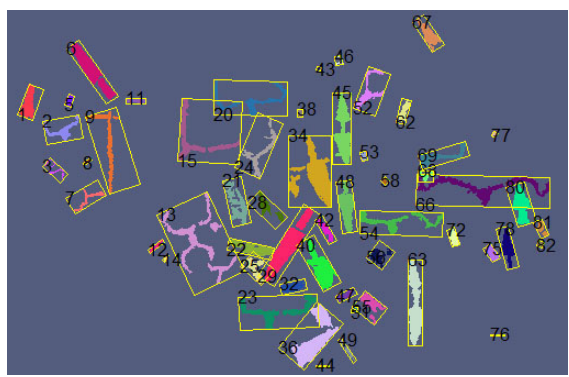

(b)

Fig. 3. Connected components recognized in step 2 of the process (a), and the clusters constructed by exploiting the EGFD distance (b)

\subsection{Dataset}

The dataset is composed of 53 scenes extracted from rock panels like the one shown in Figure 2. Some of the elements in the scenes do not have a specific form and may be classified as unrecognizable. In general, $45 \%$ of shapes in the scenes have been classified in this way.

Table 1 summarizes the statistics on the considered dataset. On average each image contains 22 symbols, 6 split symbols, and 4 connected symbols.

Table 1. Statistics on the dataset considered in the experiments

\begin{tabular}{|c|c|c|c|}
\hline \# Scenes & \# Symbols & $\begin{array}{c}\text { \#connected } \\
\text { Symbols }\end{array}$ & $\begin{array}{c}\text { \# split } \\
\text { Symbols }\end{array}$ \\
\hline 53 & 1215 & 330 & 249 \\
\hline
\end{tabular}

In order to perform our experiments we created a dataset of petroglyphs collected by de Lumley's team on Mount Bego and published in [4]. The basic dataset consists of 94 images from 10 classes.

\subsection{Experiment Setup}

The experiment was performed on a Windows 7 notebook with an Intel $i 5$ processor and $4 \mathrm{G}$ RAM. The algorithm was coded in Matlab ${ }^{\circledR}$ and executed in a $2012 \mathrm{~b}$ environment. We set a threshold of 9 pixels to determine the clusters in step 3 of the algorithm. Moreover, the GFD algorithm uses 4 radial frequencies and 9 angular frequencies to index the shape.

\subsection{Results and Discussion}

In order to get a comparison basis, the GFD algorithm was applied to each component of the scenes obtaining the following results. Among the recognizable 
shapes (54\%), GFD correctly recognized $55 \%$ of them. The application of the proposed algorithm increased the recognition rate of $10 \%$.

Figure 4 provides statistics on the clustering algorithm for each class symbol. In particular, the purple bars indicate the percentage of each class symbol, i.e., the antropomorphic symbols represent about $4 \%$ of petroglyphs in the scenes. The red and brown bars indicate the percentage of correctly clustered symbols that have been recognized and unrecognized, respectively. For example, about $24 \%$ of the generated clusters regarded antropomorphic symbols and about $13 \%$ of them were correctly recognized.

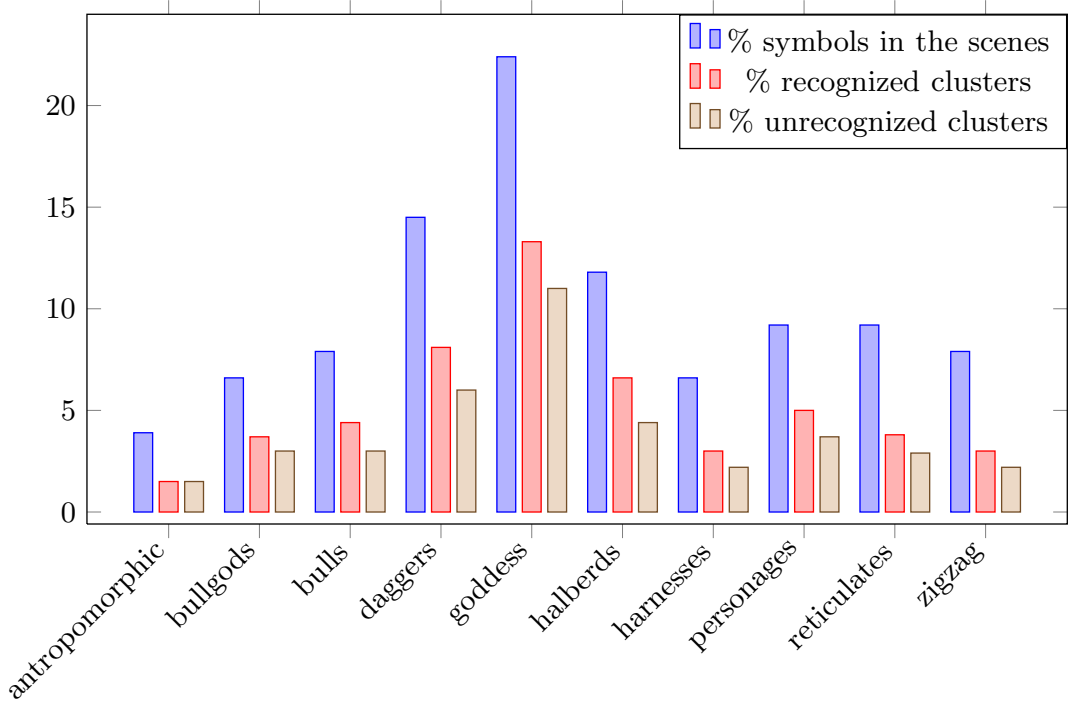

Fig. 4. Percentage of symbol classes in the scenes, percentage of well clustered and recognized symbols, percentage of well clustered but not recognized symbols

To highlight the quality of the proposed approach we compared our results with those achieved by using GHT [21] and IDM [7] descriptors on the same dataset. GHT is an image processing technique for arbitrary shape detection [1], while IDM is a distance robust to local distortions [10.

Table 2 provides the statistics of their performance in terms of recognition rate and speed. It is worth to note that since IDM and GHT are not rotation invariant, in the experiment we rotate the query image by 90,180 , and 270 degrees. The results highlight that EGFD outperforms the other approaches both in terms of recognition rate and time execution. IDM is able to obtain similar recognition results due to their robustness to deformation, but it is time expensive to be computed. On the other hand, GHT obtains good performances with respect to IDM but bad recognition rates. 
Table 2. Comparison among EGFD, IDM, and GHT descriptors in terms of recognition rate and time performance

\begin{tabular}{|c|c|c|}
\hline Image Descriptor & Recognition rate & Time \\
\hline EGFD & $65 \%$ & $1.3 \mathrm{secs}$ \\
\hline IDM $^{*}$ & $61 \%$ & $76 \mathrm{secs}$ \\
\hline GHT $^{*}$ & $46 \%$ & $6.28 \mathrm{secs}$ \\
\hline
\end{tabular}

*applied to the query image rotated at $0,90,180$, and 270 degrees.

\section{Conclusion and Future Work}

In this paper we have presented a recognition algorithm based on the Generic Fourier Descriptor able to identify shapes extracted from petroglyph scenes. This descriptor is useful because its ability to be fast and independent from rotation. The latter characteristic is particularly important because petroglyphs are used to present in many different positions, scales, and angles. This algorithm was associated to different modules which clean and decompose scenes, eliminating noises derived by rock cracks and other not significant elements, and isolate and recompose the parts in all the possible ways for evaluating the most similar petroglyph of the dataset. To evaluate the efficacy of the algorithm we carried out an experiment taking into account the petroglyphs and the scenes found in Mount Bego. The outcomes of the experiment are encouraging also considering the chaotic set of symbols. We have also compared the proposed GFD-based algorithm with Image Deformation Model (IDM) 10] and Generalized Hough Transform (GHT) 21] descriptors obtaining better performances both in terms of recognition accuracy and time.

In the future we plan to delivery the recognizer as mobile application supporting the use of query by sketch to ease user interaction [28] and improve the performances through the indexing of the petroglyph database [13].

Acknowledgement. This research is supported by the "Indiana MAS and the Digital Preservation of Rock Carvings: A multi-agent system for drawing and natural language understanding aimed at preserving rock carvings" FIRB project funded by the Italian Ministry for Education, University and Research, under grant RBFR10PEIT.

\section{References}

1. Ballard, D.H.: Generalizing the hough transform to detect arbitrary shapes. Pattern Recognition 13(2), 111-122 (1981)

2. Costagliola, G., Deufemia, V., Polese, G., Risi, M.: A parsing technique for sketch recognition systems. In: Proc. of Symp. on VLHCC, pp. 19-26 (2004)

3. Costagliola, G., Polese, G.: Extended positional grammars. In: Proc. Int'l Conf. on Visual Languages, pp. 103-110 (2000) 
4. de Lumley, H., Echassoux, A.: The rock carvings of the chalcolithic and ancient bronze age from the mont bego area. The Cosmogonic Myths of the Early Metallurgic Settlers in the Southern Alps. L'Anthropologie 113(5), 969-1004 (2009)

5. Deufemia, V., Paolino, L., de Lumley, H.: Petroglyph recognition using selforganizing maps and fuzzy visual language parsing. In: Proc. of IEEE Int'l Conf. on Tools with Artificial Intelligence (ICTAI 2012), pp. 852-859 (2012)

6. Deufemia, V., Paolino, L., Tortora, G., Traverso, A., Mascardi, V., Ancona, M., Martelli, M., Bianchi, N., De Lumley, H.: Investigative analysis across documents and drawings: Visual analytics for archaeologists. In: Proc. of Int'l Working Conf. on Advanced Visual Interfaces, pp. 539-546 (2012)

7. Deufemia, V., Paolino, L.: Combining unsupervised clustering with a non-linear deformation model for efficient petroglyph recognition. In: Bebis, G., et al. (eds.) ISVC 2013, Part II. LNCS, vol. 8034, pp. 128-137. Springer, Heidelberg (2013)

8. Deufemia, V., Risi, M., Tortora, G.: Sketched symbol recognition using latentdynamic conditional random fields and distance-based clustering. Pattern Recognition 47(3), 1159-1171 (2014)

9. Echassoux, A., de Lumley, H., Pecker, J.C., Rocher, P.: Les gravures rupestres des Plaiades de la montagne sacre du Bego, Tende, Alpes-Maritimes, France. Comptes Rendus Palevol 8(5), 461-469 (2009)

10. Keysers, D., Deselaers, T., Gollan, C., Ney, H.: Deformation models for image recognition. IEEE Trans. on PAMI 29, 1422-1435 (2007)

11. Mascardi, V., Briola, D., Locoro, A., Grignani, D., Deufemia, V., Paolino, L., Bianchi, N., de Lumley, H., Malafronte, D., Ricciarelli, A.: A holonic multi-agent system for sketch, image and text interpretation in the rock art domain. Int'l Journal of Innovative Computing, Information and Control 10(1), 81-100 (2014)

12. Mascardi, V., Deufemia, V., Malafronte, D., Ricciarelli, A., Bianchi, N., de Lumley, H.: Rock art interpretation within indiana MAS. In: Jezic, G., Kusek, M., Nguyen, N.-T., Howlett, R.J., Jain, L.C. (eds.) KES-AMSTA 2012. LNCS, vol. 7327, pp. 271-281. Springer, Heidelberg (2012)

13. Nappi, M., Polese, G., Tortora, G.: First: Fractal indexing and retrieval system for image databases. Image and Vision Computing 16(14), 1019-1031 (1998)

14. Seidl, M., Breiteneder, C.: Detection and Classification of Petroglyphs in Gigapixel Images - Preliminary Results. In: Proc. of VAST, pp. 45-48 (2011)

15. Seidl, M., Breiteneder, C.: Automated petroglyph image segmentation with interactive classifier fusion. In: Proc. of the Indian Conf. on Computer Vision, Graphics and Image Processing, pp. 66:1-66:8 (2012)

16. Sher, Y.A.: Petroglyphs in Central Asia. Nauka (1980)

17. Takaki, R., Toriwaki, J., Mizuno, S., Izuhara, R., Khudjanazarov, M., Reutova, M.: Shape analysis of petroglyphs in central asia. Forma 21, 91-127 (2006)

18. Zhang, D., Lu, G.: Enhanced generic fourier descriptors for object-based image retrieval. In: Proc. of ICASSP, vol. 4, pp. 3668-3671 (2002)

19. Zhang, D., Lu, G.: Shape based image retrieval using generic fourier descriptors. Signal Processing: Image Communication 17, 825-848 (2002)

20. Zhang, J., Kang, K., Liu, D., Yuan, Y., Yanli, E.: Vis4heritage: Visual analytics approach on grotto wall painting degradations. IEEE Transactions on Visualization and Computer Graphics 19(12), 1982-1991 (2013)

21. Zhu, Q., Wang, X., Keogh, E., Lee, S.H.: An efficient and effective similarity measure to enable data mining of petroglyphs. Data Min. Knowl. Discov. 23(1), 91-127 (2011) 